ORIGINAL ARTICLE

\title{
Relation between isokinetic muscle strength and functional capacity in recreational athletes with chondromalacia patellae
}

\author{
Y Yildiz, T Aydin, U Sekir, C Cetin, F Ors, T Alp Kalyon
}

Br J Sports Med 2003;37:475-479

See end of article for authors' affiliations .....................

Correspondence to: Dr Yildiz, Gulhane Askeri Tip Akademisi, Department of Sports Medicine, Etlik, Ankara 06018, Turkey; yasabis@yahoo.com

Accepted 22 January 2003

\begin{abstract}
Objectives: To investigate the effects of isokinetic exercise on pain and functional test scores of recreational athletes with chondromalacia patellae (CMP) and to examine the correlation between isokinetic parameters and functional tests or pain score.

Methods: The functional ability of 30 recreational athletes with unilateral CMP was evaluated using six different tests. Pain scores were assessed during daily activities before and after the treatment protocol. Isokinetic exercise sessions were carried out at angular velocities of $60 \% \mathrm{~s}\left(25-90^{\circ}\right.$ range of flexion) and $180 \%$ (full range). These sessions were repeated three times a week for six weeks.

Results: Quadriceps and hamstring peak torque, total work, and endurance ratios had improved significantly after the treatment, as did the functional parameters and pain scores. There was a poor correlation between the extensor endurance ratio and one leg standing test. A moderate correlation between the visual analogue scale and the extensor endurance ratio or flexion endurance ratio was also found.

Conclusions: The isokinetic exercise programme used in this study had a positive effect on muscle strength, pain score, and functional ability of knees with CMP. The improvement in the functional capacity did not correlate with the isokinetic parameters.
\end{abstract}

$\mathrm{T}$ he knee joint is often exposed to sports injury because of its function in various activities and its limited motion. Patellofemoral disorders are some of the most commonly treated conditions. Anterior knee pain may result from articular cartilage damage, retinacular tightness, patellofemoral malalignment, localised trauma, or inflammation of periarticular soft tissue. A systematic approach to the assessment of anterior knee pain is critical for accurate diagnosis and initiation of the correct treatment. ${ }^{1-3}$ Chondromalacia patellae (CMP) is evaluated in cases of patellofemoral pain syndrome. ${ }^{4}$ The term is used to describe pathological lesions of the patellar articular cartilage found at magnetic resonance imaging, arthroscopy, or arthrotomy. ${ }^{5}$ The patellofemoral joint is a complex articulation because of its dependence on both dynamic and static restraints for stability. ${ }^{67}$

Patellofemoral pain is typically activity induced and aggravated by actions that increase patellofemoral compressive forces, such as ascending and descending stairs, inclined walking, squatting, and prolonged sitting. ${ }^{8}$

CMP and other patellofemoral disorders are often accompanied by patellofemoral instability. This can be simply a reflex inhibition of the quadriceps muscle secondary to pain. ${ }^{8}$ In this respect, a significant correlation between isokinetic parameters or pain and functional tests would be expected. However, the relation between functional performance tests and isokinetic muscle strength is controversial, and the general opinion is that there is poor correlation.

Patellofemoral instability is not only related to mechanical factors but also to proprioceptive deficits caused by mechanoreceptor injury. ${ }^{8}$ Rehabilitation must therefore focus on restoring the proprioceptive mechanism by enhancing cognitive appreciation of the respective joint relative to position and movement, and by providing muscular stabilisation of the joint in the absence of structural restraints. Irrgang and Pezzullo ${ }^{9}$ have stated that exercise has a positive influence on articular nutrition because of compression of the articular surfaces and decreased oedema. Thus, when developing an exercise programme, it is important to keep in mind the biomechanics of the joint and the possible deleterious effects of inappropriate exercise. The patellofemoral compressive forces are reduced as the testing speed is increased. ${ }^{10}$

It has been shown that isokinetic exercises can improve muscle endurance and power. The aim of this study was to determine the effects of isokinetic training on the muscular strength and endurance, pain, and functional status of recreational athletes with CMP. We investigated the relations between the isokinetic parameters, functional capacity, and pain scores.

\section{MATERIALS AND METHODS Subjects}

Thirty male recreational athletes (mean (SD) age 24 (4) years, height $173(5) \mathrm{cm}$, weight $73.0(6.2) \mathrm{kg}$, body fat percentage $22.1(4.3)$ ) were recruited. All subjects had unilateral CMP diagnosed by magnetic resonance imaging. They had no contralateral lower extremity pathologies, neurological problems, or other conditions that could be aggravated by the testing protocol or could confound the test results. None had undertaken an exercise programme within the preceding three months. They were requested to refrain from unusual activities or vigorous exercise for 24 hours before their testing session.

Magnetic resonance imaging was performed with a $1.5 \mathrm{~T}$ magnet (Magnetom VISION; Siemens, Erlargen, Germany), a circular polarised knee coil (Siemens), and a fat suppressed gradient echo sequence (FLASH-3D, fast low angle shot;

Abbreviations: CMP, chondromalacia patellae; VAS, visual analogue scale; VMO, vastus medialis obliquus 
repetition time $(\mathrm{TR})=45$ milliseconds; echo time $(\mathrm{TE})=$ 11 milliseconds; flip angle $=30^{\circ}$; imaging time $=14$ minutes). Sagittal images were obtained with a section thickness of $2 \mathrm{~mm}$ and an in plane resolution of $0.31 \times 0.31 \mathrm{~mm}^{2}$, field of view (FOV) of $16 \mathrm{~cm}$, and matrix of $512 \times 512$ pixels. All images were transferred to a multiprocessing workstation (Octane Duo; Silicon Graphics Inc, Mountain View, California, USA).

\section{Isokinetic measurement}

Isokinetic dynamometry was performed to evaluate quadriceps and hamstring peak torque and work strength. The contralateral knees were used as controls. Maximal concentric force was measured by determining maximal concentric force moment (peak torque) during flexion and extension. The Cybex dynamometer was calibrated as part of the regular schedule for maintenance of equipment used for this testing device. ${ }^{11}$

The knee to be tested was placed on the knee flexionextension plate of the Cybex Norm device, according to the manufacturer's instructions for isolating knee flexion and knee extension, and was secured with Velcro straps. ${ }^{11}{ }^{12}$ The length of the dynamometer was adapted to the length of the knee of each subject. To familiarise themselves with the testing device, subjects were instructed to perform three active repetitions of knee movement ranging from maximal flexion to maximal extension. Standard stabilisation strapping was placed across the distal thigh and chest, and placements were limited to grasping the waist stabilisation strap. Before the testing session started, the subject was allowed a 10 minute warm up at a light intensity (less than $50 \mathrm{~W}$ ) on a cycle ergometer, followed by a 30 second stretch of the quadriceps and hamstring muscles. Selection of the extremity was random. The same investigator performed all the tests. Subjects were instructed to give $100 \%$ effort and received positive feedback during testing. They were allowed three submaximal contractions of the quadriceps and hamstring muscle group at the beginning of the test condition to familiarise themselves with the test conditions. They were given five maximal contractions at $60 \%$ and 15 maximal contractions at $180 \%$ for each test condition. The best peak torque and power contraction of the five and 15 test contractions for each test condition were collected for data analysis. Between each condition, the subjects were allowed to rest for one minute and gravitational corrections were performed.

\section{Functional ability of the knee}

We evaluated the functional ability of the knee using six different tests. The reliability and validity of these tests have been demonstrated in several studies. The tests performed were: the one leg standing test; the single limb hopping course; the one legged hop for distance; the triple legged hop for distance; the six metre hop for time (seconds); the cross six metre hop for time (seconds).

\section{Single limb hopping course}

This test is especially useful for documenting ankle function on an uneven surface. ${ }^{14}$ The jumping course consists of eight squares, four of which are even, one square has a $15^{\circ}$ increase, another square has a $15^{\circ}$ decrease, and two squares show a $15^{\circ}$ lateral incline. The volunteers are asked to jump across the course on one leg, touching each area once, as fast as possible without leaving the course. The test result is quantified as the time taken in seconds to complete the course. For each failure, an extra second is added to the time taken to complete the course.

\section{One leg standing test}

This test evaluates ability to balance while standing on one leg. ${ }^{13-15}$ The subject is asked to stand on one leg for one minute with eyes open and for another minute with eyes closed to exclude visual perception. In contrast with current thinking, we performed this test not on a hard surface but on a soft surface to increase the failure rate. Each surface contact with the contralateral leg was counted as one failure point.

\section{One legged and triple legged hop for distance}

Patients were asked to make one and three forceful hopping movements forward as large as they could. The distance between the starting point and the end point was measured. Two tests were performed, and the average distance was measured for each test.

\section{Six metre and cross six metre hop for time}

This is a timed test performed over a distance of $6 \mathrm{~m}$. Each subject was encouraged to use linear, large, forceful one legged hopping movements and crosswise, large, forceful one legged hopping movements to propel his body the measured distance. Two tests were performed and the average time was measured for each test.

\section{Pain assessment}

A visual analogue scale (VAS) was used to score pain during daily activities before and after the treatment protocol.

\section{Isokinetic exercise protocol}

The Cybex Norm isokinetic system was used for the isokinetic exercise programme. Each exercise session was carried out with three settings of 10 repetitions at $60 \%$ and 20 repetitions at $180^{\circ} \mathrm{s}$. The isokinetic exercise over the $25-90^{\circ}$ range of flexion at $60 \%$ angular velocity was chosen to minimise patellofemoral compression force and not to provoke pain. These sessions were carried out three times a week for six weeks.

\section{Data analysis}

The Cybex NORM software program provided the measures of peak torque, standardised peak torque (peak torque divided by body weight), power, and standardised power (power divided by body weight). Descriptive statistics of the means, standard deviations, and ranges were determined for each subject. Student's $t$ test was performed for measurements obtained before and after treatment. The Pearson product-moment correlation test was performed between the percentage change in isokinetic muscle parameters and functional tests. The 0.05 probability level was accepted as significant. All values are given as mean (SD).

\section{RESULTS}

Tables 1 and 2 give descriptive statistics of each measurement for all subjects. Student's $t$ test was used to determine if there was any significant difference between values obtained before and after the isokinetic exercise programme.

Mean peak torque at $60 \%$ angular velocity and mean total work and endurance at $180 \%$ angular velocity in extension and flexion activities were significantly different after the isokinetic exercise programme (table 1, fig 1). There also was a significant difference in the values for the one leg standing test and single limb hopping course $(p=0.0001)$. A significant difference was found in all the functional test values (table 2). VAS used to assess pain improved from 5.8 to 1.2.

\section{Correlation analysis}

The percentage change in values was used for correlation analysis. The isokinetic data did not correlate with the functional parameters except for the percentage change in 
Table 1 Isokinetic exercise effects on muscle values

\begin{tabular}{lllll}
\hline Knee EX/FX & & Before & After & p Value \\
\hline EXPT (N.m) & CMP & $135(49)$ & $169(54)$ & 0.0001 \\
& Control & $159(25)$ & $162(26)$ & 0.062 \\
FXPT (N.m) & CMP & $75(30)$ & $101(38)$ & 0.0001 \\
& Control & $93(22)$ & $96(24)$ & 0.203 \\
EXTW (J) & CMP & $105(33)$ & $130(42)$ & 0.0001 \\
& Control & $112(30)$ & $114(31)$ & 0.072 \\
FXTW (J) & CMP & $64(27)$ & $85(35)$ & 0.0001 \\
& Control & $70(21)$ & $71(20)$ & 0.167 \\
EXEN & CMP & $99(13)$ & $87(12)$ & 0.0001 \\
& Control & $83(16)$ & $81(17)$ & 0.072 \\
FXEN & CMP & $105(19)$ & $88(16)$ & 0.0001 \\
& Control & $88(16)$ & $86(17)$ & 0.072 \\
\hline
\end{tabular}

CMP, chondromalacia patellae; EX, Extension; FX, flexion; PT, peak torque at $60 \%$ angular velocity; TW, total work at $180 \%$ s angular velocity; $\mathrm{EN}$, endurance.

extensor endurance ratios and the percentage change in one legged standing tests $(r=-41 ; \mathrm{p}=0.025)$. We also found a moderately significant correlation between the percentage change in VAS and extensor endurance ratios $(r=-52$; $\mathrm{p}=0.005)$ and the percentage change in VAS and flexor endurance ratios $(r=-45 ; \mathrm{p}=0.013)$.

\section{DISCUSSION}

Knowledge of the mechanics of a joint is critical to design programmes for its rehabilitation. The vastus medialis obliquus (VMO) is aruably the most important muscle in patellar mechanics. ${ }^{1}$ Weakness of this muscle can cause the patella to displace laterally, resulting in an increase in patellofemoral compression forces. ${ }^{16-18}$ Patellofemoral stress is defined by the formula: stress $=$ force/area. In a closed chain model (leg press or squatting), the joint reaction force on the patellofemoral joint increases as the knee flexes from 0 to $90^{\circ}$. The contact area also increases, but the change is less than for the force. Therefore stress increases as the knee flexes from 0 to $90^{\circ}$. Closed chain exercises are safest in the $0-45^{\circ}$ range. In contrast, in the open chain model (leg curls and knee extension), exercises are most safely carried out in the $25-90^{\circ}$ range. ${ }^{19-21}$ Irrgang and Pezzullo ${ }^{9}$ have stated that exercise has a positive influence on articular nutrition because of compression of the articular surfaces and decreased oedema. Thus it is important to keep in mind the biomechanics of the joint and the possible deleterious effects of inappropriate exercise when developing programmes. In this study, the first eight sessions of the isokinetic exercise were performed at the terminal knee extension $\left(0-30^{\circ}\right.$ of extension) to minimise patellofemoral joint reaction force and so as not to provoke pain. However, this protocol can be in conflict with open kinetic chain exercise. ${ }^{22}$ In our study, isokinetic exercise at an angular speed of $60 \%$ to improve maximal quadriceps muscle force and $25-90^{\circ}$ range of flexion was chosen to reduce reaction forces on the patellofemoral joint. The compressive forces are reduced as the testing speed is increased. Therefore a full range of motion was selected at $180 \%$ s to improve muscle endurance. ${ }^{10}$

In recent years, isokinetic exercises have commonly been used in sports medicine practice and have increased muscle conditioning and reduced clinical complaints. ${ }^{123}$ Furthermore, their effectiveness for patellofemoral pain syndrome has been confirmed. It is well known that there is an increase in proprioceptive ability after muscular rehabilitation protocols. There are specific exercise programmes to improve muscle strength and knee functional capacity. However, an ideal exercise programme should improve not only muscular stability but also functional capacity. The effects of isokinetic exercises on functional
Table 2 Effects of isokinetic exercise on functional test scores

\begin{tabular}{lllll}
\hline Stability tests & & Before & After & p Value \\
\hline OLHD $(\mathrm{cm})$ & CMP & $104(32)$ & $118(39)$ & 0.0001 \\
& Control & $123(38)$ & $132(40)$ & 0.0001 \\
TLHD $(\mathrm{cm})$ & CMP & $368(118)$ & $403(127)$ & 0.0001 \\
& Control & $380(124)$ & $414(121)$ & 0.0001 \\
CSMHT (s) & CMP & $3.5(1.6)$ & $2.8(1.3)$ & 0.0001 \\
& Control & $3.3(1.1)$ & $2.9(0.9)$ & 0.0001 \\
CSMHT (s) & CMP & $5.7(4.5)$ & $4.7(3.0)$ & 0.005 \\
& Control & $4.3(2.0)$ & $3.8(1.5)$ & 0.0001 \\
OLST (s) & CMP & $18.1(8.8)$ & $14.5(7.8)$ & 0.0001 \\
& Control & $14.7(7.2)$ & $11.5(6.8)$ & 0.0001 \\
SLHC (s) & CMP & $16.1(7.1)$ & $13.4(5.0)$ & 0.0001 \\
& Control & $14.6(5.7)$ & $11.9(3.9)$ & 0.0001 \\
\hline
\end{tabular}

CMP, Chondromalacia patellae; OLST, one leg standing test; SLHC, single limb hopping course; OLHD, one legged hop for distance; TLHD, triple legged hop for distance; SMHT, six metre hop for time (seconds); CSMHT, cross six metre hop for time (seconds).

capacity and muscular strength in patients with CMP have not previously been studied.

The VMO is the most important muscle in patellar mechanics. ${ }^{1}$ The imbalance between the vastus medialis and lateralis muscles is usually regarded as the main cause of patellofemoral pain syndrome. Therefore the main target of rehabilitation protocols for CMP is the vastus medialis muscle. It has been impossible to predict the effect of isokinetic exercises on the vastus medialis only. As assessment of isolated vastus medialis and vastus lateralis strength in vivo is not possible, electromyography has been used to compare the relative recruitment of these muscles with the rationale that decreased activity of the VMO relative to the vastus lateralis is indicative of compromised medial patellar stability. Some authors have found significant differences in VMO and vastus lateralis activity in patients with patellofemoral pain ${ }^{24-26}$ but others have not. ${ }^{27-30}$ Direct comparisons of these studies are difficult because of differences in experimental techniques and methods of assessing electromyographic data.

It has been found that isolated vastus medialis contractions could not be achieved by isokinetic exercises. ${ }^{131}$ The isokinetic exercise programme of our study is different, so we need to verify the contribution of the vastus medialis by electromyographic studies.

CMP causes restriction in daily activities such as stair climbing and squatting. In these activities the medial extensor muscle support is extremely important. In this study, significant improvements were found in peak torque $(p=0.0001)$, total work $(p=0.0001)$, and endurance of the knee extensors. The improvement in the strength of this muscle group also led to improvement in the clinical status of the athletes. In addition, the torque, work, and endurance

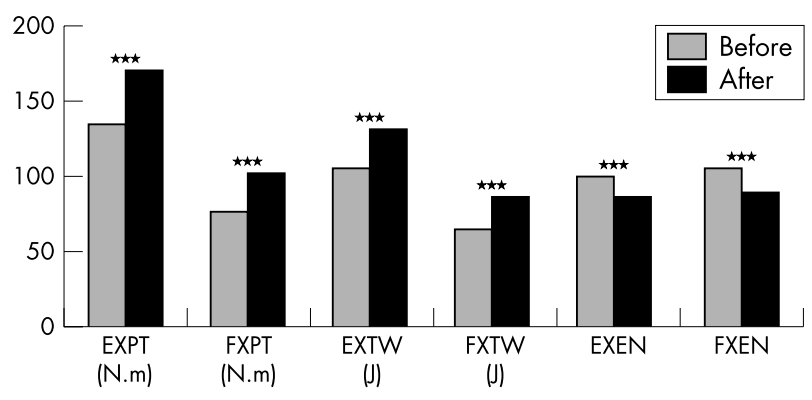

Figure 1 Effects of isokinetic exercise programme on muscle values. EX, Extension; FX, flexion; PT, peak torque at $60 \%$ angular velocity; TW, total work at $180 \%$ s angular velocity; $E N$, endurance. ${ }^{* *} \mathrm{p}=0.0001$. 
values of the flexor muscles were significantly improved after the isokinetic exercise protocol (table 1, fig 1). These findings confirm the efficacy of these isokinetic exercises in the rehabilitation of recreational athletes with CMP.

In our study, the VAS score was significantly decreased after the isokinetic exercise programme. Patellofemoral compression forces have also been found to be reduced after isokinetic exercises..$^{32}$ It has been shown that the reduction in patellofemoral compression forces may lead to a decrease in nociceptor activity. ${ }^{33}$ The pathophysiology of CMP is not clearly understood. The most commonly accepted hypothesis is related to abnormal patellar tracking which increases patellofemoral joint stress and subsequent articular cartilage wear. Although articular cartilage is aneural and has been dismissed as a possible source of symptoms, it has been proposed that the subadjacent endplate is exposed to pressure variation that would normally be absorbed by healthy cartilage..$^{35}$ This mechanical stress is believed to stimulate pain receptors in the subchondral bone. Powers et $a l^{37}$ suggested that locomotor function in patients with patellofemoral pain is associated with increased quadriceps femoris muscle torque, lending support to the concept of strengthening as a useful treatment option. Despite these findings, however, the mechanism by which strengthening improves patellofemoral pain symptoms and functional ability has not been established.

It is possible that gross quadriceps strengthening alters the contact location and pressure distribution, possibly relieving a sensitive area. In this study, quadriceps peak torque and total work increased from 135 N.m and 105 J to 169 N.m and $135 \mathrm{~J}$ respectively $(\mathrm{p}=0.0001)$. The quadriceps endurance ratio improved from 99 to $87(\mathrm{p}=0.0001)$. Therefore the isokinetic exercise programme increased the muscle endurance ratios and peak torque. This resulted from a decrease in the patellofemoral compressive forces and relief of the sensitive area.

\section{Functional tests}

These tests are performed to evaluate muscular strength, balance, proprioception, and coordination of complex movement patterns. Balance can be assessed with the single leg stance test. ${ }^{38} 39$ Significant differences were found between the functional test values before and after the isokinetic exercise programme (table 2). As can be seen, the isokinetic exercises positively affected the functional test scores of not only the knees with CMP but also the healthy knees. This therefore shows that isokinetic exercise programmes can be used to increase the performance of athletes, as they have positive effects on complex movement patterns.

Some studies have found deficits in neuromuscular joint stabilisation after joint pathologies. This results from partial deafferentation caused by the damage to the joint mechanoreceptors. This is also responsible for chronic joint pathologies. ${ }^{12} 4041$ The aim of proprioceptive rehabilitation is to increase muscular stabilisation of the joints, in order to develop joint perception and position sense.

The one leg standing test and single limb hopping course give us some information about the proprioceptive state. In our study, the one leg standing test improved from 18.1 to $14.5(\mathrm{p}=0.0001)$ and the single limb hopping course decreased from 16.1 to 13.4 seconds $(p=0.0001)$. These results may show that the proprioceptive deficit can be normalised by isokinetic exercise.

CMP and other patellofemoral disorders are often accompanied by patellofemoral instability. Patellofemoral instability is not only related to mechanical factors but also to proprioceptive deficits caused by mechanoreceptor injury. ${ }^{8}$ This instability can be simply a reflex inhibition of the quadriceps muscle secondary to pain. ${ }^{8}$ From this point of view, a significant correlation between isokinetic parameters, pain, and functional tests is expected. However, the relation between functional performance tests and isokinetic muscle strength is controversial, and the general opinion is that there is poor correlation. If there were a relation, we could interpret the functional capacity and pain scores in CMP. We did not find any relation between change in peak torque at $60 \%$ or change in total work at $180 \%$ and functional test scores. This may be related to the non-functional nature of isokinetic exercises. On the other hand, there was a poor correlation between the percentage change in extensor endurance ratios and the percentage change in the values for the one legged standing test $(r=-41 ; \mathrm{p}=0.025)$. This agrees with findings in the literature.

A VAS was used to score pain during daily activities before and after the exercise protocol. A moderately significant correlation was observed between the change in VAS and the change in extensor or flexor endurance ratio. The first result was higher than the second. This finding may show that isokinetic exercise can minimise pain by decreasing the patellofemoral compression forces, thus increasing the endurance of the extensor muscle group. On the other hand, it may only indicate that isokinetic exercise improves the endurance of the extensor muscle group.

\section{Conclusion}

The knee with CMP has reduced muscular strength and functional capacity. The isokinetic exercise programme used in this study had a positive effect on the functional ability of the knee and muscle strength, endurance ratio, and work capacity. Thus this protocol can be used for rehabilitation in CMP. The VAS may be helpful in assessing the muscle endurance of recreational athletes with CMP. It has been observed that isokinetic tests are not sufficient to evaluate functional capacity. Therefore functional tests should be applied in patients with CMP to assess functional capacity.

\section{Authors' affiliations}

Y Yildiz, T Aydin, U Sekir, C Cetin, T Alp Kalyon, Gulhane Askeri Tip Akademisi, Department of Sports Medicine, Etlik, Ankara 06018, Turkey F Ors, Gulhane Askeri Tip Akademisi, Department of Radiodiagnostic Radiology, Etlik, Ankara

\section{REFERENCES}

1 Powers CM. Rehabilitation of patellofemoral joint disorders: a critical review. J Orthop Sports Phys Ther 1998;28:345-54.

2 Kannus $\mathbf{P}$, Aho $H$, Jarvinen $M$, et al. Computerized recording of visits to an outpatient sports clinic. Am J Sports Med 1987;15:79-85.

3 Milgrom C, Finestone A, Eldad A, et al. Patellofemoral pain caused by overactivity. J Bone Joint Surg [Am] 1991;73:1041-3.

4 Arrol B, Ellis-Pegler E, Edwards A, et al. Patellofemoral pain syndrome; a critical review of the clinical trials on nonoperative therapy. Am J Sports Med 1997:25:207-12

5 Mühlbaver R, Lukasz S, Faber S, et al. Comparison of knee joint cartilage thickness in triathletes and physically inactive volunteers based on magnetic resonance imaging and three-dimensional analysis. Am J Sport Med 2000;28:541-6.

6 Walsh WM. Patellofemoral joint. In: DeLee JC, Drez D, ed. Orthopaedic sports medicine: principles and practice. Philadelphia: WB Saunders Company, 1996;2:1 163-248

7 Walsh WM, Fulkerson JP. Diagnosis and treatment of patients with patellofemoral pain. Am J Sports Med 2002;30:447-56.

8 Holmes SW, Clancy WG. Clinical classification of patellofemoral pain and dysfunction. J Orthop Sports Phys Ther 1998;28:299-306.

9 Irrgang JJ, Pezzullo D. Rehabilitation following surgical procedures to address articular cartilage lesions in the knee. J Orthop Sports Phys Ther 1998:28:232-40.

10 Mangine RE, Eifert-Mangine M, Burch D, et al. Postoperative management of the patellofemoral patient. J Orthop Sports Phys Ther 1998;28:323-34.

11 Ronkonkoma NY. Cybex Norm Int Inc: tesing and rehabilitation system: pattern selection and set up: automated protocols. User's guide. New York: Blue Sky Software Corporation, 1995.

12 Aydin T, Yildiz Y, Yildiz C, et al. Proprioception of the ankle: a comparison between female teenaged gymnasts and controls. Foot Ankle Int 2002;23:123-9. 
13 Baier M, Hopf T. Ankle ortheses effect on single-limb standing balance in athletes with functional ankle instability. Arch Phys Med Rehabil 1998;79:939-44.

14 Jerosch J, Bischof M. Proprioceptive capabilities of ankle in stable and unstable joints. Sports Exercise and Injury 1996;2:167-71.

15 Rozzi SL, Lephart SM, Sterner R. Balance training for persons with functional unstable ankles. J Orthop Sports Phys Ther 1999;29:478-86.

16 Nissen CW, Cullen MC, Hewett TE, et al. Physical and arthroscopic examination techniques of the patellofemoral joint. J Orthop Sports Phys Ther 1998;28:277-85

17 Owings TM, Grabiner MD. Motor control of the vastus medialis obliquus and vastus lateralis muscles is disrupted during eccentric contractions in subjects with patellofemoral pain. Am J Sports Med 2002;30:483-7.

18 Powers CM, Landel R, Perry J. Timing and intensity of vastus muscle activity during functional activities in subjects with and without patellofemoral pain. Phys Ther 1996;76:946-67.

19 Steinkamp LA, Dilingham MF, Markel MD, et al. Biomechanical considerations in patellofemoral joint rehabilitation. Am J Sports Med 1993;21:438-44.

20 Stiene HA, Brosky T, Reinking MF, et al. A comparison of closed kinetic chain and isokinetic joint isolation exercise in patients with patellofemoral dysfunction. J Orthop Sports Phys Ther 1996;24:136-41.

21 Grelsamer RP, Klein JR. The biomechanics of the patellofemoral joint. J Orthop Sports Phys Ther 1998;28:286-98.

22 Alaca R, Yilmaz B, Goktepe AS, et al. Efficacy of isokinetic exercise on functional capacity and pain in patellofemoral pain syndrome. Am J Phys Med Rehabil 2002;81:807-13.

23 Douris PC. The effect of isokinetic exercise on the relationship between blood lactate and muscle fatigue. J Orthop Sports Phys Ther 1993;17:31-5.

24 Mariani PP, Caruso I. An electromyographic investigation of subluxation of the patella. J Bone Joint Surg [Br] 1979;61:169-71.

25 Souza DR, Gross MT. Comparison of vastus medialis obliquus : vastus lateralis muscle integrated electromyographic ratios between healthy subjects and patients with patellofemoral pain. Phys Ther 1991;71:310-16.

26 Wise HH, Fiebert IM, Kates JL. EMG biofeedback as treatment for patellofemoral pain syndrome. J Orthop Sports Phys Ther 1984;6:95-103.

27 Boucher JP, King MA, Lefebvre R, et al. Quadriceps femoris muscle activity in patellofemoral pain syndrome. Am J Sports Med 1992;20:527-32.
28 Maclntyre DL, Robertson GE. Quadriceps muscle activity in women runners with and without patellofemoral pain sydrome. Arch Phys Med Rehabil 1992;73:10-14.

29 Moller BN, Krebs B, Tidemand-Dal C, et al. Isometric contractions in the patellofemoral pain sydrome: an electromyographic study. Arch Orthop Trauma Surg 1976; 105:24-7.

30 Wild JJ, Franklin TD, Woods GW. Patellar pain and quadriceps rehabilitation. An EMG study. Am J Sports Med 1982;10:12-15.

31 Mirzabeigi E, Jordan C, Gronley JK, et al. Isolation of the vastus medialis obliquus muscle during exercise. Am J Sports Med 1999;27:50-3.

32 Kaufman KR, An KN, Litchy WJ, et al. Dynamic joint forces during knee isokinetic exercise. Am J Sports Med 1991;19:305-16.

33 Borsook D, LeBel AA, McPeec B. The Massachusetts General Hospital handbook of pain management. Boston, New York, Toronto, London: Little, Brown and Company, 1996

34 Wall PD. The effect of peripheral injury on cell in the spinal cord. In: Wall PD, Melzack R, eds. Textbook of pain. London: Churchill-Livingstone, 1989:11-14.

35 Sanchis-Alfonso V, Rosello-Sastre E. Immunohistochemical analysis for neural markers of the lateral retinaculum in patients with isolated symptomatic patellofemoral malalignment. Am J Sports Med 2000;28:725-31.

36 Sanchis-Alfonso V, Rosello-Sastre E, Martinez-Sanjuan V. Pathogenesis of anterior knee pain syndrome and functional patellofemoral instability in the active young. Am J Knee Surg 1999;12:29-40.

37 Powers CM, Perry J, Hsu A, et al. Are patellofemoral pain and quadriceps strength associated with locomotor function. Phys Ther 1997;77:1063-74.

38 Bohannon RW, Larkin PA, Cook AC, et al. Decrease in timed balance test scores with aging. Phys Ther 1984;64:1067-70.

39 Janda V, Vavrova M. Sensory motor stimulation. In: Liebenson C, ed. Spinal rehabilitation: a manual of active care procedures. Baltimore: Williams and Wilkins, 1996.

40 Lephart SM, Fu FH. The role of proprioception in the treatment of sports injuries. Sports Exercise and Injury 1995;1:96-02.

41 Lephart SM, Pincivero DM, Giraldo JL. The role of proprioception in the management and rehabilitation of athletic injuries. Am J Sports Med 1997:25:130-7.

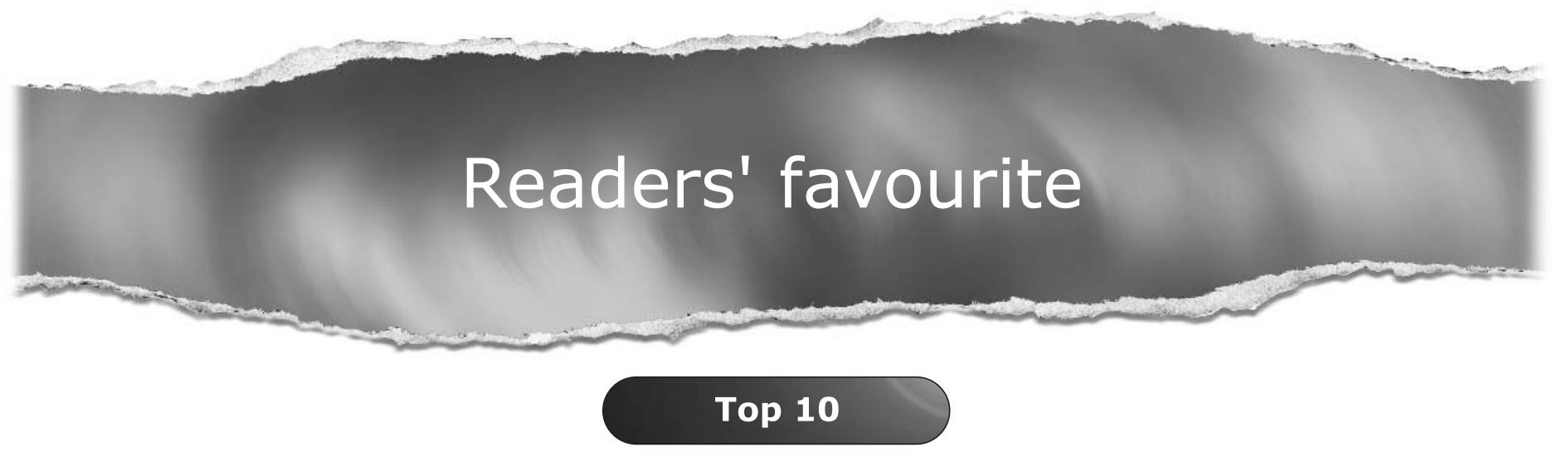

Click on the "Top 10" button on the homepage to see which are the best read articles each month

www.bjsportmed.com 